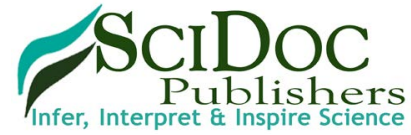

\section{Pulmonary Artery Denervation for Treatment of Pulmonary Arterial Hypertension: Results From a Controlled Before and After Study}

Research Article

Chen $\mathrm{SL}^{1 *}$, Zhang $\mathrm{H}^{2}$, Xie $\mathrm{DJ}^{2}$, Zhang $\mathrm{FF}^{2}$, Zhou $\mathrm{L}^{2}$, Zhang $\mathrm{J}^{1}$, Chen $\mathrm{MX}^{3,4}$, Stone $\mathrm{GW}^{3,4}$

${ }^{1}$ Department of Cardiology, Nanjing First Hospital, Nanjing Medical University, China.

${ }^{2}$ Department of Cardiology, Nanjing Heart Center, China.

${ }^{3}$ Emory Arts and Science College, Atlanta, USA.

${ }^{4}$ Department of Cardiology, Columbia University Medical Center, NY, USA.

\title{
Abstract
}

Background: Pulmonary arterial hypertension (PAH) is a severe disease. 6-minute walk distance (6MWD) is correlated with the prognosis of PAH patients. We previously reported the safety and efficacy of pulmonary artery denervation (PADN) for patients with idiopathic PAH (IPAH) who were unresponsive to target drugs. However, PADN has not been tested in a well-designed study for patients with IPAH/PAH or pulmonary hypertension (PH).

Objectives: The present study aimed to analyze the difference in 6-minute walk distance (6MWD) at 6-month after PADN and standard pharmacotherapy.

Methods: Between February 2013 and June 2014, a total of 28 patients with IPAH (n=11), PH from left heart disease $(\mathrm{n}=8)$, and 9 secondary PAH were included in this study. A wash-out consisting of 5 half-lives for target drugs was performed for all the patients. Next, medications were prescribed for a 6-month duration for the patients (Medication treatment). A second wash-out (5 half-lives) was completed for all the patients after the 6-month medication treatment. Finally, PADN was performed for these patients (PADN treatment), and an additional 6-month follow-up period was completed. The primary endpoint was the difference in the $\triangle 6 \mathrm{MWD}$ (defined as value at 6-month minus baseline) between two treatments.

Results: After 6-month treatment, there was significant difference in the $\triangle 6 \mathrm{MWD}$ between PADN $(65 \pm 85 \mathrm{~m})$ and Medication $(13 \pm 24 \mathrm{~m})$ treatment $(95 \% \mathrm{CI}-21.34-3.49, \mathrm{p}=0.002)$, coupling with the differences in the reduction of systolic pulmonary artery pressure (PAP, $-13.75 \pm 14.1 \mathrm{mmHg}$ vs. $-0.46 \pm 3.47 \mathrm{mmHg}, \mathrm{p}<0.001)$, mean PAP $(-7.86 \pm 6.10 \mathrm{mmHg}$ vs. $-0.14 \pm 2.48 \mathrm{mmHg}, \mathrm{p}<0.001)$, mean right atrial pressure $(-2.53 \pm 3.75 \mathrm{mmHg}$ vs. $-0.21 \pm 2.62 \mathrm{mmHg}, \mathrm{p}=0.007)$, and pulmonary vessel resistance $(-4.59 \pm 7.06$ Woods unit vs. $-0.17 \pm 2.56$ Woods unit, $\mathrm{p}=0.001)$. PADN treatment was associated with less frequent 6-month PAH-related event $(10.8 \%)$, compared to $42.9 \%$ in Medication treatment $(\mathrm{p}=0.005)$.

Conclusion: The present study showed that PADN treatment was associated with significant improvement of 6MWD and less PAH-related event. Further study was required to show the benefit of PADN in reducing mortality in patients with IPAH and secondary PAH.

Keywords: Pulmonary Arterial Hypertension; Pulmonary Artery Denervation; 6-Minute Walk Distance; Controlled Before and After Study.

Abbreviations: PAH: Pulmonary Arterial Hypertension; 6MWD: 6-Minute Walk Distance; sPAP: Systolic Pulmonary Arterial Pressure; mPAP: Mean Pulmonary Arterial Pressure; PADN: Pulmonary Artery Denervation; PVR: Pulmonary Vessel Resistance; CO: Cardiac Output; RAP: Right Atrial Pressure; RHC: Right Heart Catheterization; NT-pro BNP: N Terminal-Pro Brain Natriuretic Peptide

*Corresponding Author:

Dr. Shao-Liang Chen M.D.

Nanjing First Hospital, Nanjing Medical University, Nanjing, China.

E-mail: chmengx@126.com.

Received: August 26, 2016

Accepted: September 23, 2016

Published: September 27, 2016

Citation: Chen SL, Zhang H, Xie DJ, Zhang FF, Zhou L, et al., (2016) Pulmonary Artery Denervation for Treatment of Pulmonary Arterial Hypertension: Results From a Controlled Before and After Study. Int J Cardiol Res. 3(2), 68-77. doi: http://dx.doi.org/10.19070/2470-4563-1600012

Copyright: Chen $\mathrm{SL}^{\circ}$ 2016. This is an open-access article distributed under the terms of the Creative Commons Attribution License, which permits unrestricted use, distribution and reproduction in any medium, pro/vided the original author and source are credited. 


\section{Introduction}

Pulmonary arterial hypertension (PAH) is a severe disease characterized by progressive right ventricular (RV) failure resulting in premature death [1]. Based on the WHO classification, PAH is classified into 5 groups [1,2] based on the different mechanisms and etiologies involved in the development of PAH. Target drugs included endothelin receptor antagonists, prostacyclin, and phosphodiesterase type 5 inhibitors (5'-PDE), which have all been extensively used and confirmed to be effective and safe for $\mathrm{PAH}$ patients [1, 2]. Nevertheless, there is tremendous controversy regarding the long-term benefits and cost-effectiveness of these drugs [3]. The main reason is that the pathologic mechanisms of PAH is still under clear.

Over-activation of sympathetic nerves activity has been proposed to participate in the development of PAH [4], but medication using non-selective $\beta$-blockers did not show any improvement of PAH [5]. Based on the earlier report by Jurastch et al., [6] that pulmonary arterial (PA) sympathetic nerves ending localized around the distal PA bifurcation area and that surgical denervation of these nerves was associated with significant reduction of pulmonary arterial pressure (PAP), our group performed an actual experimental study and found that percutaneous pulmonary artery denervation (PADN) could completely abolish the increase of pulmonary arterial pressure (PAP) induced by an inflated balloon at proximal left interloba artery, the finding confirmed that PA sympathetic nerves might localize proximally [7]. Consequently, we designed our first-in-man "PADN-1 study" and results showed the benefits of PADN for idiopathic PAH (IPAH) patients who were unresponsive to target drugs [8]. However, the uncontrolled feature of the PADN-1 study [8] raised global interests and concerns regarding its safety and efficacy [9]. As a result, we designed this controlled PADN-2 study in an attempt to elucidate the improvements in a 6-minute walk distance (6MWD), as well as the hemodynamic and clinical outcomes after the PADN procedure in patients with PAH due to variety of underlying etiologies.

\section{Methods}

\section{Patient Population}

This study was conducted at two centers. Patients with a resting mean PAP $(\mathrm{mPAP}) \geq 25 \mathrm{mmHg}$ with WHO functional class IIIV PAH were included. For patients with pulmonary hypertension $(\mathrm{PH})$ induced by LHD, additional requirements included a PVR $>2.5$ woods unit and a pulmonary arterial obstructive pressure $(\mathrm{PAOP})>15 \mathrm{mmHg}$ at rest. Patients who had active inflammation and cancer were excluded. Patients with PAH secondary from portable hypertension and drug or toxin exposure were also excluded. The study protocol was approved by the Institute Research Board (Nanjing Medical University) and informal written consent was obtained from all the patients.

\section{Study Design}

The present study was designed as a controlled before and after study (Figure 1). All patients who met the inclusion and exclusion criteria were included and entered into the first wash-out period (defined as stopping all medications for at least 5 half-lives, with the exception of warfarin) as right heart catheterization and adenosine test for all patients were not performed before the study. If the patients were taking multiple drugs, the longest half-lives of the drug was selected. For example, for a patient who was taking bosentan (half-time $<5 \mathrm{~h}$ ) and digoxin (half-time $=33 \mathrm{~h}$ ), the washout period would be $5 \times 33 \mathrm{~h}=165 \mathrm{~h}$ (7 days). After the wash-

Figure 1. Study FlowChart

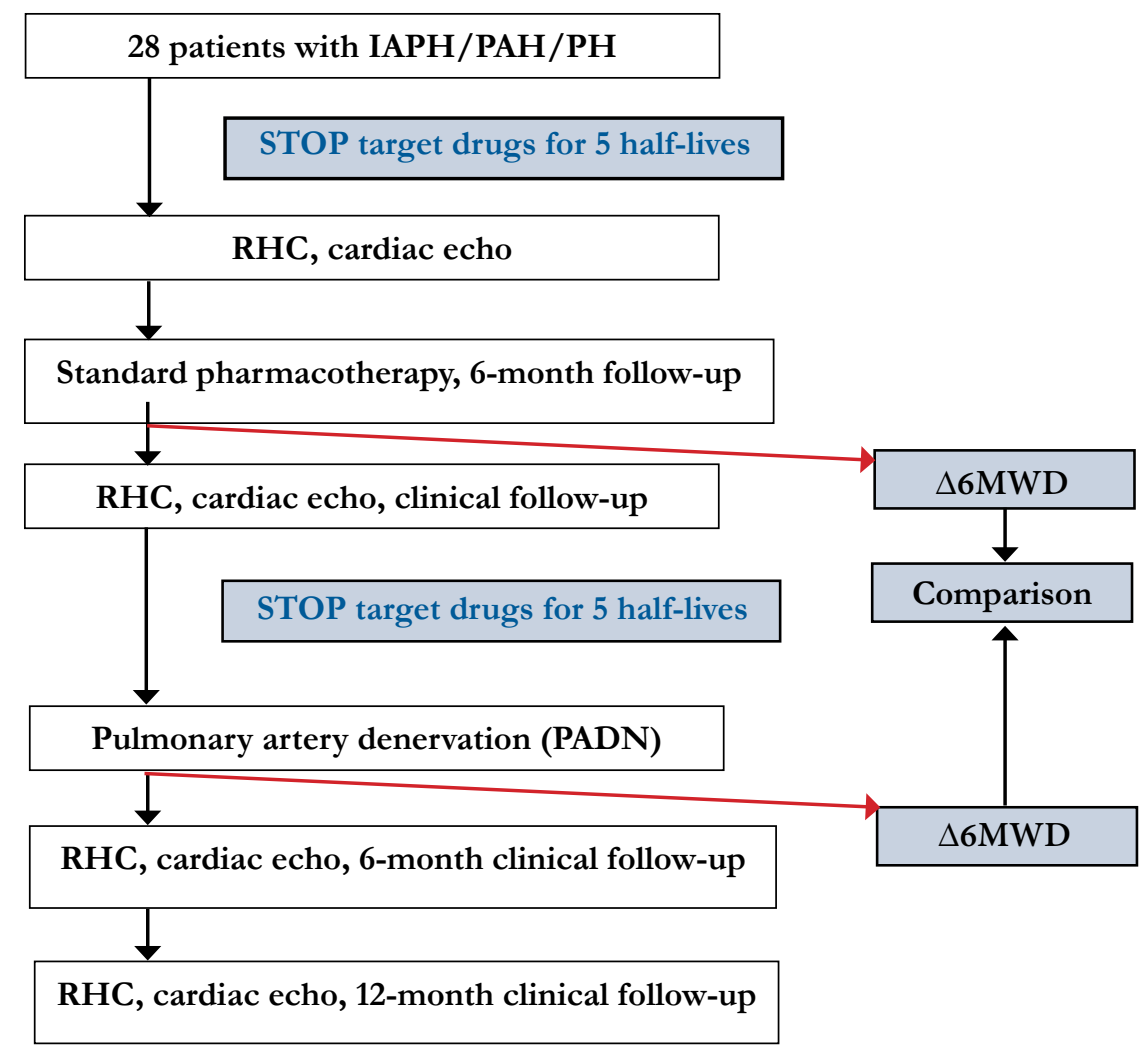


out period, the drugs were prescribed again and continued for 6 months (Medication treatment). The selection of drugs was left to the physician's discretion based on their comprehensive analysis. After 6 months, the patients underwent a second wash-out period of 5 half-lives in order to establish PADN as a standard alone therapy; next, PADN was performed, followed by 6-month and 12-month clinical follow-up (PADN treatment).

\section{Medication}

Immediately after the grouping, warfarin was continuously prescribed. Otherwise, aspirin $(100 \mathrm{mg} / \mathrm{d})$ and Plavix $(75 \mathrm{mg} / \mathrm{d})$ were prescribed instead of warfarin for patients who could not tolerate warfarin. Immediately after the PADN procedure, the standard medication for PAH were stopped for all the patients, and this was evaluated by the physician based on the patients' symptoms.

\section{Assessment of Functional Capacity}

Baseline blood samples were obtained for the analysis of N-terminal brain natriuretic peptide (NT-pro BNP) levels prior to the exercise test. The 6MWD [8] according to standard methods, the Borg scale and the WHO functional class [1] at rest and during exercise were estimated and recorded by one physician who was blinded to the study design.

\section{Echocardiographic Measurements}

All echocardiograms were performed (Vivid 7, General Electric Co., Easton Turnpike, CT, US) and interpreted in the Nanjing Echocardiographic Laboratory using the American Society of Echocardiography recommendations [10]. Digital echocardiographic data that contained a minimum of 3 consecutive beats (or 5 beats in cases of atrial fibrillation) were acquired and recorded. RV systolic pressure (sRVP) is equal to systolic PAP (sPAP) in the absence of pulmonary stenosis. SPAP is equal to the sum of right atrial (RA) pressure (RAP) and the RV to RA pressure gradient during systole. RAP was estimated based on the echocardiographic features of the inferior vena cava and was assigned a standard value [11]. The RV to RA pressure gradient was calculated as $4 v_{t}^{2}$ using the modified Bernoulli equation in which $\mathrm{v}_{t}$ is the velocity of the tricuspid regurgitation jet in $\mathrm{m} / \mathrm{s}$. mPAP was estimated ac- cording to the velocity of the pulmonary regurgitation jet in $\mathrm{m} / \mathrm{s}$. The tricuspid excursion index (Tei) [12] is defined as (A - B)/B in which $A$ is the time interval between the end and the onset of tricuspid annular diastolic velocity, and B is the duration of tricuspid annular systolic velocity (or the RV ejection time).

\section{Right Heart Catheterization}

A 7F flow-directed Swan-Ganz catheter (Edwards, USA) was inserted percutaneously into the internal jugular vein for the measurements of the resting RAP, sRVP, sPAP, mPAP, PAOP, and cardiac output $(\mathrm{CO})$ values. The PVR [= (mPAP-PAOP $) / \mathrm{CO}]$ was then calculated. All the measurements were performed at the end of expiration. If the PAOP measurement was unreliable, the left ventricular end-diastolic pressure was measured and used rather than the PAOP measurement. Two blood samples from the RA, RV and PA were obtained for the measurements of oxygen pressure and saturation. If the difference between the oxygen pressure or saturation measurement of these two samples was $>7 \%$, further sampling was performed to identify the location of the left-to-right shunt.

\section{PADN Procedure}

PADN was performed at three sites (A, B and C) at the conjunctional area between the distal main trunk and the ostial left branch (Figure 2). The following ablation parameters were programmed at each point: a temperature of $45^{\circ} \mathrm{C}-50^{\circ} \mathrm{C}$, energy $\leq 15 \mathrm{~W}$, and a time of 120 seconds. The procedure would cease for 10 seconds if the patient felt intolerable chest pain during the procedure. The EKG and pressure lines (including cardiac output) were monitored and continuously recorded throughout the procedure.

The success of a PADN procedure was defined as a reduction of sPAP or mPAP immediately after the procedure, or at 24 hours with a $\geq 10 \%$ compared to the baseline values, without intra-procedural complications.

\section{Study Endpoints}

The primary endpoint was the difference in 6MWD after the 6-month between the medication and PADN treatment. The sec-

Figure 2. (I) anterior-posterior and cranial $\left(20^{\circ}\right)$ view of pulmonary arterial angiograph.

(II) red line represents the lateral wall of MPA, blue for anterior wall of LPA, the crossing site by these two lines is the point A; the crossing site by yellow line (reflects the posterior wall of LPA) and red line is the point B which is 1-2 mm posteriori to the point A; green line starts from the inferior wall of RPA and ends at the point A, the point $C$ localizes at this level and 1-2 mm anteriorly to the point $A$

(III) a PADN catheter with 10 electrodes is positioned at the distal MPA, electrodes A, B and C match with the points A, Band C.
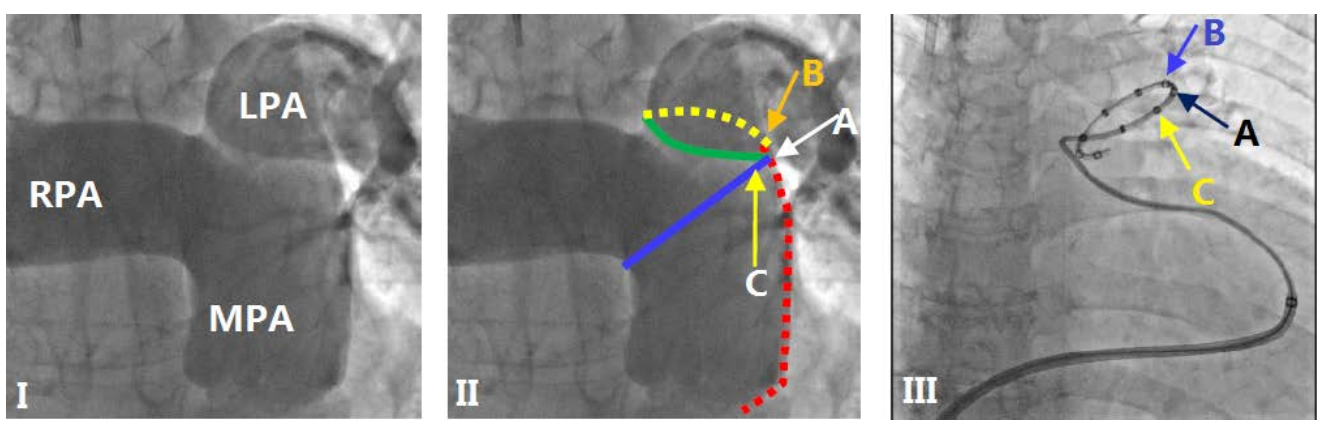
ondary endpoints included composite and individual PAH-related events, including the worsening of $\mathrm{PAH}$, the initiation of treatment with the intravenous or subcutaneous injection of drugs, lung transplantation, atrial septostomy or all-cause death. Repeat hospitalization also served as a secondary endpoint.

\section{Follow-Up}

The patients were monitored in the CCU for at least $24 \mathrm{~h}$. All measurements were repeated post-procedure, at $24 \mathrm{~h}$, at 3 -month, at 6-month, and at 12-month. CT scanning of the pulmonary artery were performed before and at 6-month after the PADN procedure.

\section{Definition and Outcome Measures}

The worsening of PAH was defined as the occurrence of all three of the following measurements: a decrease in the 6MWD of at least $15 \%$ from baseline, confirmed by a second 6MWD performed on a different day within 14 days of the first measurement; a worsening of the symptoms of PAH; and the need for additional treatment for PAH. A worsening of the symptoms of PAH was defined as any of the following measurements: a change from baseline to a higher WHO functional class (or no change in patients who were in WHO functional class IV at baseline) and the appearance or worsening of signs of right heart failure, which did not respond to oral diuretic therapy. An independent clinical event committee adjudicated, in a blind fashion, all the events related to PAH and all the deaths that were reported up to the end of the treatment.

\section{Statistical Analysis}

All the analyses were performed using the statistical program SPSS 19.0 (SPSS Institute Inc., Chicago, IL., USA). Statistical significance was defined as a two-sided $\mathrm{P}$ value $<0.05$. Based on our previous data, the $\triangle 6 \mathrm{MWD}$ (defined as the 6MWD at the 6-month follow-up minus the baseline 6MWD) was approximately $+60 \mathrm{~m}$ after PADN treatment and $+15 \mathrm{~m}$ after the medication treatment. A total of 28 patients were required to achieve significance (2-sided p-value, $80 \%$ power) between the two arms. The difference in each variable (at the 6-month period minus the baseline) for each treatment was calculated and compared between the two treatments. The continuous variables were expressed as the mean \pm SD. A normality test for all the continuous variables was performed using the Kolmogorov-Smirnov and Shapiro-Wilk tests. The differences in the continuous variables between the two treatments were analyzed using a paired $t$-test or the MannWhitney $U$ test when appropriate. The categorical variables were compared using Fisher's exact test. The event-free survival rate was generated using the Kaplan-Meier method and was analyzed with the log-rank test.

\section{Results}

\section{Baseline Patient Characteristics}

Between March 2013 and June 2013, a total of 28 patients (11 males and 17 females, with an average age of 49 years) were enrolled in the current study. Within this cohort, there were 8 patients with idiopathic PAH (IPAH), 9 patients with pulmonary hypertension (PH) induced by left heart dysfunction (LHD), and 11 PAHs secondary to CTD ( $\mathrm{n}=4$, connective tissue disease), chronic thromboemboli $(n=3$, CTE) and congenital heart disease after surgical repair ( $n=4$, CHDSR, Table 1$)$, respectively. The mean time interval from the diagnosis of $\mathrm{PAH}$ to the present study was 4.24 years. At enrollment, most of the patients were put on diuretics and prostacyclins; an endothelin receptor antagonist was prescribed for 5 IPAH patients, and all the LHD patients received a beta-blocker treatment.

\section{Medical Treatment}

After the first wash-out for 5 half-lives, $21.4 \%$ of patients were put on prostacyclin, and an endothelin receptor antagonist was prescribed to $35.8 \%$ of patients (Table 1). During the second wash-out, there was 1 patient with previous myocardial infarction who is medically dependent on diuretics and this was prescribed immediately.

\section{Improvement of 6MWD Between Treatments}

At 6-month, the NT-pro BNP and WHO functional class decreased significantly after both treatments (Table 2). Notably, the 6MWD increased from $361 \pm 112 \mathrm{~m}$ to $373 \pm 111 \mathrm{~m}$ in the Medication treatment $(\mathrm{p}=0.009)$ and from $358 \pm 115 \mathrm{~m}$ to $423 \pm 98$ $\mathrm{m}$ in the PADN treatment $(\mathrm{p}<0.001)$. The 6MWD after PADN treatment, but neither before any treatment nor after medication, was negatively correlated with $\operatorname{mPAP}(\mathrm{r}=-0.416, \mathrm{p}=0.028)$ and $\operatorname{sPAP}(\mathrm{r}=-0.401, \mathrm{p}=0.034$, Figure $3 \mathrm{~A})$, and WHO functional class $(\mathrm{r}=-0.710, \mathrm{p}<0.001)$ and PVR $(\mathrm{r}=-0.380, \mathrm{p}=0.046)$.

The $\Delta 6 \mathrm{MWD}$ was $+13 \pm 24 \mathrm{~m}(+3.9 \%$ increase) 6 -month after Medication treatment, which was significantly different to the $65 \pm 85 \mathrm{~m}$ measurement $(+23.9 \%$ increase, 95\% CI: $-21.34 \sim$ 3.49, $\mathrm{p}=0.002$, Table 3) 6-month after PADN treatment. After Medication treatment, there were 9 patients $(32 \%)$ whose 6MWD decreased (range from $-6 \mathrm{~m}$ to $-47 \mathrm{~m}$ ). Of those 9 patients, 5 patients had an average 6MWD increase of $45 \mathrm{~m} 6$ months after the PADN treatment, whereas no change was observed in 4 patients. When follow-up was extended to one-year, there were 2 patients $(7 \%)$ after the PADN procedures who had no changes in 6MWD. At one-year after PADN procedure, the average $\triangle 6 \mathrm{MWD}$ was $92 \mathrm{~m}$ (Figure 3B).

\section{Comparison of the Differences in Hemodynamic Between the Two Treatments}

The $\Delta$ sPAP, $\Delta$ mPAP, $\Delta$ sRVP and $\Delta$ mean RAP values measured by either cardiac echo or right heart catheterization at 6-month after PADN treatment were greater than those at 6-month after Medication treatment (all $\mathrm{p}<0.05$, Table 3), with resultant significant differences in the increase of cardiac output $\left(+0.67 \pm 1.05 \mathrm{~L} / \mathrm{min} / 1.73 \mathrm{~m} 2\right.$ vs. $+0.06 \pm 0.24 \mathrm{~L} / \mathrm{min} / 1.73 \mathrm{~m}^{2}$, $95 \%$ CI: $-0.10 \sim-0.20, \mathrm{p}=0.005)$, reduction of the pericardial fluid amount $(-0.74 \pm 2.63 \mathrm{~mm}$ vs. $+0.11 \pm 0.93 \mathrm{~mm}, 95 \% \mathrm{CI}$ : $-1.04 \sim 2.47, \mathrm{p}=0.036)$ and Tei $(-0.34 \pm 0.11$ points vs. $-0.04 \pm 0.09$ points, 95\% CI: 0.24 0.35, $\mathrm{p}<0.001)$. At one-year after PADN treatment, there was a gradual reduction of either mPAP or PVR (Figure 3C).

All the measurements were non-significant different between patients with secondary $\mathrm{PH}$ from left heart disease and patients 
Table 1. Baseline Characteristics and Medical Treatment.

\begin{tabular}{|c|c|}
\hline Variables & Results \\
\hline Patient number, $\mathrm{n}$ & 28 \\
\hline Male, $\mathrm{n}(\%)$ & $11(39.3)$ \\
\hline Age, yr & $49 \pm 17$ \\
\hline \multicolumn{2}{|l|}{ Etiology, n (\%) } \\
\hline IPAH & $11(39.3)$ \\
\hline LHD & $8(28.6)$ \\
\hline CTD & $2(7.1)$ \\
\hline CTEPH & $3(10.7)$ \\
\hline CHDSR & $4(14.3)$ \\
\hline Time from diagnosis of PAH to screening, yr & $4.24 \pm 7.13$ \\
\hline Chest pain & $8(28.6)$ \\
\hline Syncope & $4(14.3)$ \\
\hline Fatigue & $27(96.4)$ \\
\hline Dyspnea & $27(96.4)$ \\
\hline \multicolumn{2}{|c|}{ Medication, $\mathrm{n}(\%)$ at screening } \\
\hline Diuretics & $23(82.1)$ \\
\hline Calcium-channel antagonist & $4(14.3)$ \\
\hline Beta-blocker & $8(28.6)$ \\
\hline Prostacyclin & $23(82.1)$ \\
\hline 5'-PDE & $10(35.7)$ \\
\hline ET receptor antagonist & $5(17.9)$ \\
\hline Digoxin & $13(46.4)$ \\
\hline \multicolumn{2}{|l|}{ Medication treatment* } \\
\hline Diuretics & $22(78.6)$ \\
\hline Calcium-channel antagonist & $7(25.0)$ \\
\hline Beta-blocker & $8(28.6)$ \\
\hline Prostacyclin & $6(21.4)$ \\
\hline 5'PDE & $11(39.3)$ \\
\hline ET receptor antagonist & $10(35.8)$ \\
\hline Digoxin & $10(35.7)$ \\
\hline
\end{tabular}

*indicated the medication started immediately after first wash-out

IPAH: Idiopathic Pulmonary Hypertension; LHD: Left Heart Disease; CTD: Connective Tissue Disease; CTEPH: Chronic Thrombolyticpulmonary Hypertension; CHDSR: Congenital Heart Disease After Surgical Repair; 5’-PDE: Phosphodiesterase Type 5 Inhibitor.

Table 2. Comparison of Measurements Between Medication and PADN Treatment.

\begin{tabular}{|c|c|c|c|c|c|c|}
\hline & \multicolumn{3}{|c|}{ Medication $(n=28)$} & \multicolumn{3}{|c|}{$\operatorname{PADN}(\mathrm{n}=28)$} \\
\hline & Prior-to & 6-month & $\mathrm{p}$ & Prior-to & 6-month & $\mathrm{p}$ \\
\hline NT-pro BNP, pg/ml & $2293 \pm 2741$ & $1732 \pm 1878$ & 0.007 & $2669 \pm 3178$ & $1296 \pm 947$ & 0.015 \\
\hline WHO class, point & $2.75 \pm 0.52$ & $2.46 \pm 0.58$ & 0.009 & $2.75 \pm 0.52$ & $2.21 \pm 0.63$ & $<0.001$ \\
\hline 6MWD, mm & $361 \pm 112$ & $373 \pm 111$ & 0.009 & $358 \pm 115$ & $423 \pm 98$ & $<0.001$ \\
\hline Cardiac output, L/min & $3.2 \pm 0.94$ & $3.26 \pm 0.89$ & 0.221 & $3.25 \pm 1.05$ & $3.91 \pm 1.08$ & 0.002 \\
\hline \multicolumn{7}{|c|}{ RHC } \\
\hline sPAP, mmHg & $91.9 \pm 33.0$ & $91.5 \pm 33.4$ & 0.485 & $91.9 \pm 33.3$ & $78.2 \pm 29.4$ & $<0.001$ \\
\hline $\mathrm{mPAP}, \mathrm{mmHg}$ & $56.1 \pm 21.1$ & $55.9 \pm 21.2$ & 0.762 & $56.7 \pm 21.8$ & $48.9 \pm 19.2$ & $<0.001$ \\
\hline PCWP, $\mathrm{mmHg}$ & $13 \pm 7.7$ & $13.8 \pm 6.9$ & 0.365 & $12.5 \pm 8.6$ & $12.9 \pm 6.3$ & 0.788 \\
\hline mRAP, $\mathrm{mmHg}$ & $11.5 \pm 4.2$ & $11.8 \pm 4.6$ & 0.442 & $11.4 \pm 4.7$ & $8.8 \pm 3.4$ & 0.001 \\
\hline sRVP, mmHg & $89.3 \pm 31.2$ & $91.3 \pm 32.1$ & 0.255 & $89.3 \pm 31.6$ & $83.0 \pm 34.7$ & 0.147 \\
\hline PVR, woods unit & $13.8 \pm 7.6$ & $13.6 \pm 6.9$ & 0.721 & $14.3 \pm 8.6$ & $9.7 \pm 6.8$ & 0.002 \\
\hline \multicolumn{7}{|c|}{ Cardiac echo } \\
\hline $\mathrm{mPAP}, \mathrm{mmHg}$ & $47.9 \pm 24.2$ & $45.6 \pm 23.2$ & $<0.001$ & $47.4 \pm 24.8$ & $38.1 \pm 16.5$ & 0.002 \\
\hline $\mathrm{mRAP}, \mathrm{mmHg}$ & $11.8 \pm 3.1$ & $11.1 \pm 2.8$ & 0.043 & $11.8 \pm 3.1$ & $8.9 \pm 3.1$ & $<0.001$ \\
\hline sRVP, mmHg & $96.0 \pm 87.9$ & $87.9 \pm 26.9$ & $<0.001$ & $96.1 \pm 31.7$ & $78.6 \pm 23.3$ & $<0.001$ \\
\hline $\mathrm{sPAP}, \mathrm{mmHg}$ & $98.1 \pm 29.5$ & $87.2 \pm 25.2$ & $<0.001$ & $98.2 \pm 32.2$ & $79.9 \pm 24.6$ & $<0.001$ \\
\hline Pericardial fluid, mm & $1.11 \pm 1.64$ & $1.43 \pm 1.93$ & 0.059 & $1.96 \pm 2.89$ & $0.85 \pm 0.89$ & 0.002 \\
\hline RV Tei, \% & $0.59 \pm 0.17$ & $0.55 \pm 0.16$ & 0.029 & $0.69 \pm 0.09$ & $0.36 \pm 0.09$ & $<0.001$ \\
\hline
\end{tabular}

PADN: Pulmonary Artery Denervation; NT-pro BNP: N Terminal-Pro Brain Natriuretic Peptide; 6MWD: 6-Minute Walk Distance; RHC: Right Heart Catheterization; sPAP: Systolic Pulmonary Arterial Pressure; mPAP: Mean Pulmonary Arterial Pressure; mRAP: Mean Right Atrial Pressure; sRVP: Systolic Right Ventricular (RV)Pressure; PVR: Pulmonary Vessel Resistance. 
Table 3. Comparison of the Differences of Measurements After the 6-Month Treatment Between the Medication and PADN Treatment

\begin{tabular}{|c|c|c|c|c|}
\hline & Medication & PADN & 95\% CI & p \\
\hline NT-pro BNP, pg/ml & $-562 \pm 1009$ & $-1373 \pm 2792$ & $-42.84 \sim 1665.44$ & 0.062 \\
\hline $\begin{array}{c}\text { WHO functional } \\
\text { class,point }\end{array}$ & $-0.36 \pm 0.49$ & $-0.54 \pm 0.58$ & $-0.10 \sim 0.46$ & 0.202 \\
\hline 6MWD, m & $13 \pm 24$ & $65 \pm 85$ & $-21.34 \sim-3.49$ & 0.002 \\
\hline \% of increase & $3.9 \%$ & $23.9 \%$ & $-0.31 \sim-0.09$ & 0.001 \\
\hline Cardiac output, L/min & $0.06 \pm 0.24$ & $0.67 \pm 1.05$ & $-0.10 \sim-0.20$ & 0.005 \\
\hline \multicolumn{5}{|c|}{ RHC } \\
\hline sPAP, mmHg & $-0.46 \pm 3.47$ & $-13.75 \pm 14.1$ & $8.38 \sim 18.19$ & $<0.001$ \\
\hline mPAP, mmHg & $-0.14 \pm 2.48$ & $-7.86 \pm 6.10$ & $5.26 \sim 10.18$ & $<0.001$ \\
\hline PCWP, mmHg & $0.82 \pm 4.72$ & $0.36 \pm 6.96$ & $-1.63 \sim 2.56$ & 0.653 \\
\hline mRAP, mmHg & $-0.21 \pm 2.62$ & $-2.53 \pm 3.75$ & $0.70 \sim 3.93$ & 0.007 \\
\hline sRVP, mmHg & $1.96 \pm 8.93$ & $-6.22 \pm 22.07$ & $1.76 \sim 14.62$ & 0.014 \\
\hline PVR, woods unit & $-0.17 \pm 2.56$ & $-4.59 \pm 7.06$ & $1.99 \sim 6.83$ & 0.001 \\
\hline \multicolumn{5}{|c|}{ Cardiac echo } \\
\hline mPAP, mmHg & $-2.36 \pm 2.78$ & $-9.28 \pm 13.93$ & $1.98 \sim 11.86$ & 0.008 \\
\hline mRAP, mmHg & $-0.54 \pm 1.57$ & $-2.86 \pm 2.86$ & $1.20 \sim 3.44$ & $<0.001$ \\
\hline sRVP, mmHg & $-8.07 \pm 9.73$ & $-17.54 \pm 16.97$ & $4.78 \sim 14.15$ & $<0.001$ \\
\hline sPAP, mmHg & $-10.89 \pm 11.87$ & $-18.25 \pm 16.73$ & $3.13 \sim 11.58$ & 0.001 \\
\hline Pericardial fluid, mm & $0.11 \pm 0.93$ & $-0.74 \pm 2.63$ & $-1.04 \sim 2.47$ & 0.036 \\
\hline RV Tei, \% & $-0.04 \pm 0.09$ & $-0.34 \pm 0.11$ & $0.24 \sim 0.35$ & $<0.001$ \\
\hline
\end{tabular}

PADN: Pulmonary Artery Denervation; NT-pro BNP: N Terminal-Pro Brain Natriuretic Peptide; 6MWD: 6-Minute Walk Distance; RHC: Right Heart Catheterization; sPAP: Systolic Pulmonary Arterial Pressure; mPAP: Mean Pulmonary Arterial Pressure; mRAP:

Mean Right Atrial Pressure; sRVP: systolic Right Ventricular (RV)Pressure; PVR: Pulmonary Vessel Resistance

Figure 3A. Systolic pulmonary arterial pressure $(\mathrm{sPAP}, \mathrm{p}=0.034)$ and mean pulmonary arterial pressure $(\mathrm{mPAP}, \mathrm{p}=0.028)$ after $6-\mathrm{month}$ treatment were negatively correlated with with 6-minute walk distance.

Figure 3B. Dynamic change of 6-minute walk distance (6MWD). The average increment of 6MWD at 6-month and 12-month after PADN treatment was $65 \mathrm{~m}$ and $93 \mathrm{~m}$, respectively, it was $12 \mathrm{~m}$ after the 6-month medication.

Figure 3C. Gradual reduction of mean pulmonary arterial pressure (mPAP) and pulmonary vessel resistance (PVR) prior-to, 6-month after medication, and after pulmonary artery denervation (PADN).
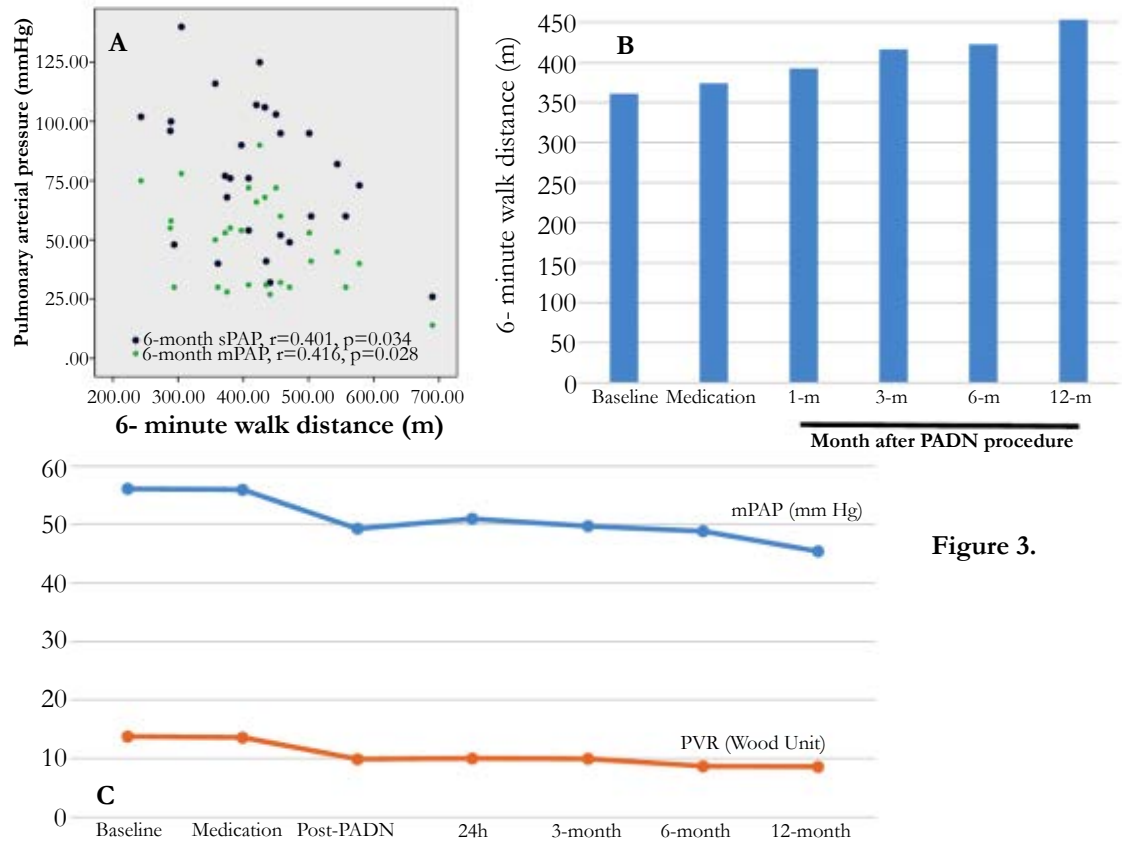
Table 4. Comparison of The Differences of Measurements at 6-Month After PADN Between IPAH/Secondary PAH And PH From Left Heart Disease.

\begin{tabular}{|c|c|c|c|c|}
\hline & IPAH/PAH & PH & $\mathbf{9 5 \%}$ CI & p \\
\hline NT-pro BNP, pg/ml & $-747 \pm 1241$ & $-2696 \pm 4467$ & $-283.7 \sim 4182.4$ & 0.084 \\
\hline WHO functional class, point & $-0.53 \pm 0.51$ & $-0.56 \pm 0.73$ & $-0.46 \sim 0.52$ & 0.903 \\
\hline 6MWD, m & $53 \pm 72$ & $89 \pm 108$ & $-106.7 \sim 33.6$ & 0.294 \\
\hline Cardiac output, L/min & $0.66 \pm 1.20$ & $0.68 \pm 0.68$ & $-0.91 \sim-0.86$ & 0.951 \\
\hline \multicolumn{5}{|c|}{ RHC } \\
\hline sPAP, mmHg & $-14.9 \pm 16.7$ & $-11.1 \pm 5.6$ & $-15.7 \sim 8.0$ & 0.511 \\
\hline mPAP, mmHg & $-8.3 \pm 6.7$ & $-6.9 \pm 4.8$ & $-6.5 \sim 3.8$ & 0.584 \\
\hline PCWP, mmHg & $1.1 \pm 6.7$ & $-1.2 \pm 7.6$ & $-3.5 \sim 8.1$ & 0.419 \\
\hline mRAP, mmHg & $-2.2 \pm 3.23$ & $-3.2 \pm 4.78$ & $-2.2 \sim 4.1$ & 0.524 \\
\hline sRVP, mmHg & $-12.8 \pm 22.2$ & $-13.5 \pm 21.1$ & $-7.4 \sim 28.9$ & 0.234 \\
\hline PVR, woods unit & $-5.3 \pm 8.4$ & $-3.2 \pm 2.7$ & $-7.9 \sim 3.9$ & 0.479 \\
\hline \multicolumn{5}{|c|}{ Cardiac echo } \\
\hline Pericardial fluid, mm & $-1.2 \pm 2.8$ & $-0.7 \pm 1.7$ & $-2.6 \sim 1.5$ & 0.596 \\
\hline RV Tei, $\%$ & $-0.33 \pm 0.09$ & $-0.35 \pm 0.14$ & $-0.07 \sim 1.11$ & 0.687 \\
\hline
\end{tabular}

PADN: Pulmonary Artery Denervation; NT-pro BNP: N Terminal-Pro Brain Natriuretic Peptide; 6MWD: 6-Minute Walk Distance; RHC: Right Heart Catheterization; sPAP: Systolic Pulmonary Arterial Pressure; mPAP: Mean Pulmonary Arterial Pressure; mRAP: Mean Right Atrial Pressure; sRVP: Systolic Right Ventricular (RV)Pressure; PVR: Pulmonary Vessel Resistance.

with IPAH and secondary PAH (Table 4).

\section{Clinical outcome after the treatment}

After the 6-month Medication treatment, a PAH-related event was observed in 12 patients (42.9\%), higher than 3 patients $(10.8 \%)$ at 6 -month after PADN treatment $(\mathrm{p}=0.002$, Figure $4 \mathrm{~A})$; these events were mainly driven by the worsening of PAH (Table 5). The mean time from treatment to clinical worsening was 125 days (range of 22 to 166 days). After medication treatment, which was significantly shorter than the 166 days (range from 47 to 172 days) reported after PADN treatment $(\mathrm{p}=0.01)$. Re-hospitalization was required in $42.9 \%$ of the patients in the Medication compared to $14.4 \%$ of the patients in the PADN treatment $(\mathrm{p}=0.018$, Table 5$)$.

There were no peri-PADN procedure access hematomas. Before PADN procedure, all patients received CT scanning of PA. At 6-month after PADN treatment, CT scanning was performed for 24 patients, and there were no signs of aneurysms and thrombus formations

At the one-year follow-up, there were $5(17.9 \%)$ PAH-related events ( 2 new events, including 2 sudden deaths). The 6MWD in patients without PAH-related events was $467 \pm 100 \mathrm{~m}$, which was higher than the $393 \pm 42 \mathrm{~m}$ in patients with PAH-related event $(p=0.018)$. When the patients were stratified according to 6MWD (cut-off value of $440 \mathrm{~m}$ ), the rate of PAH-related events in patients with $6 \mathrm{MWD} \geq 440 \mathrm{~m}$ was $7.7 \%$, compared to $26.7 \%$ $(\mathrm{p}=0.042$, Figure 4B) in patients with $6 \mathrm{MWD}<440 \mathrm{~m}$. Of the two deaths (1 CHDSR patient and 1 IPAH patient), the 6MWD values at one-year were $303 \mathrm{~m}$ and $279 \mathrm{~m}$, even with $\Delta 6 \mathrm{MWD}$ of $82 \mathrm{~m}$ and $66 \mathrm{~m}$, respectively.

The survival rate-free from PAH-related event at 6-month follow- up was $88.9 \%$ in patients with secondary $\mathrm{PH}$ from left heart disease after PADN, similar to $89.5 \%$ in patients with IPAH and secondary $\mathrm{PAH}(\mathrm{p}=0.926$, Figure $4 \mathrm{C})$.

\section{Cost analysis}

After 6-month Medication treatment, the overall cost (35000 \pm 12000 USD) was much more expensive than that $(6000 \pm 700$ USD, $\mathrm{p}<0.001)$ at 6 -month after PADN procedure.

\section{Discussion}

The major findings of this study are as follows: [1] PADN, at a much lower cost, is safe and is associated with a significant increase in 6MWD [2]; the PADN procedure led to a greater improvement of pulmonary arterial hemodynamics with subsequently less frequent PAH-related events and re-hospitalizations.

\section{MWD, Exercise Capacity and Hemodynamics}

6MWD has been selected as an endpoint in previous studies for PAH patients [13]. Notably, we found that 6MWD improvement were negatively associated with the WHO functional class, which suggests that a $15 \%$ reduction in the 6 MWD may be clinically meaningful; this result supports the use of a $15 \%$ reduction in the 6MWD as a criterion for PAH worsening in clinical studies $[14,15]$.

PAP, RAP and PVR were the three most important parameters correlated with the prognosis of the PAH patients $[1,16]$. A sPAP between $50-70 \mathrm{mmHg}$ and $\mathrm{RAP}>8 \mathrm{mmHg}$ were markers used to indicate the severity of PAH disease [17]. As a result, a more profound reduction of RAP and PAP was the goal in previous 
Table 5. Clinical Follow-Up After the 6-Month Treatment in 28 Patients.

\begin{tabular}{|c|c|c|c|}
\hline & Medication & PADN & p \\
\hline PAH-event, $\mathrm{n}(\%)$ & $12(42.9)$ & $3(10.8)$ & 0.002 \\
\hline All-cause death & 0 & 0 & \\
\hline Atrial septostomy & 0 & 0 & \\
\hline Lung transplantation & 0 & 0 & \\
\hline Needing IV \& IS & $2(7.2)$ & 0 & \\
\hline Worsening of PAH & $10(36.0)$ & $3(10.8)$ & \\
\hline Re-hospitalization, $\mathrm{n}(\%)$ & $12(38.3)$ & $4(14.4)$ & 0.018 \\
\hline Cost, x10,000USD/per pt & $3.5 \pm 1.2$ & $0.6 \pm 0.7$ & $<0.001$ \\
\hline Any-cause Death, $\mathrm{n}(\%)$ & 0 & 0 & \\
\hline Access site hematoma, $\mathrm{n}(\%)$ & 0 & 0 & \\
\hline Aneurysm, $\mathrm{n}(\%)$ & 0 & 0 & \\
\hline Thrombus, $\mathrm{n}(\%)$ & 0 & 0 & \\
\hline
\end{tabular}

PADN: Pulmonary Artery Denervation ; PAH: Pulmonary Arterial Hypertension; pt: Patient.

Figure 4A. Rate of PAH-related event was $42.9 \%$ after the 6 -month medication treatment, significantly higher than $10.7 \%$ at the 6-month after PADN procedure $(\mathrm{p}=0.005)$.

Figure 4B. Comparison of PAH-related event at one-year follow-up between patients with 6-minute walk distance $<440$ $\mathrm{m}(26.7 \%)$ and $\geq 440 \mathrm{~m}(7.7 \%, \mathrm{p}=0.044)$ after PADN treatment. PAH, pulmonary arterial hypertension; PADN, pulmonary artery denervation.

Figure 4C. Rate of PAH-related event in patients with secondary PH from left heart disease was 88.9\% at 6-month after

PADN, similar to $89.5 \%$ in patients with IPAH and secondary PAH $(p=0.926)$.
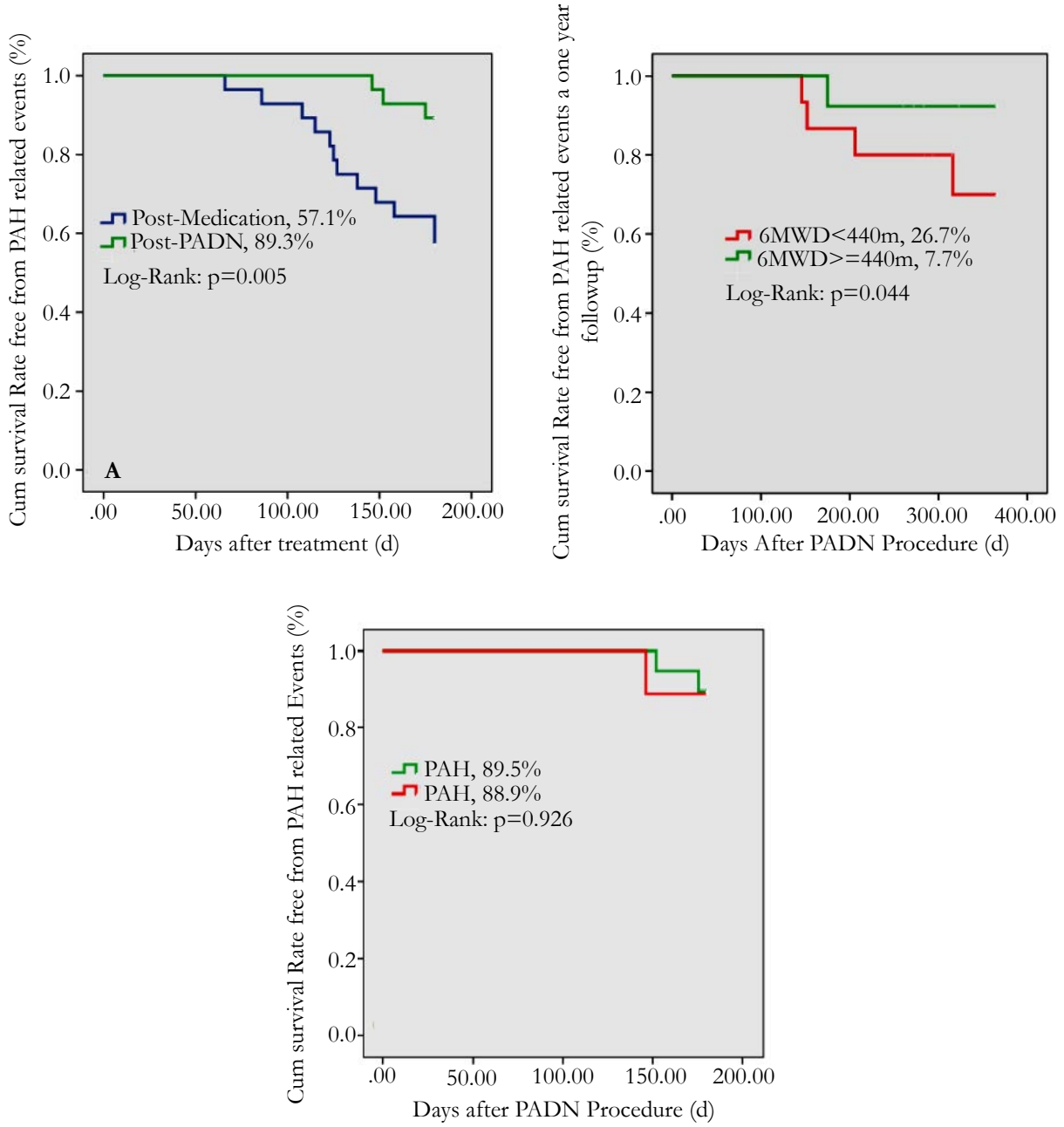
studies [16, 18-20]. We found that the 6MWD improvement after the PADN procedure was paralleled by an improvement in RAP, sPAP and mPAP. However, we failed to establish a correlation between the PA hemodynamics with 6MWD post-medication. Consistent with previous report [21], this finding suggest that this relationship requires a more significant reduction of PAP and a higher increase of $6 \mathrm{MWD}$, as we found the negative relationship between PAP and 6MWD after PADN procedure.

\section{MWD and clinical outcomes}

Some studies $[2,8,22]$ with a short-term follow-up reported a relationship between baseline 6MWD and clinical events, but two meta-analysis studies $[13,23]$ failed to demonstrate this relationship and showed that the absolute 6MWD $(\geq 440 \mathrm{~m})$ induced by treatment was associated with a lower mortality, which was supported by our results showing that patients with $6 M W D \geq 440 \mathrm{~m}$ had a lower rate of PAH-related events. Insight analysis into the correlation of 6MWD with clinical outcomes based on previous studies [11, 22-24], a short follow-up period, and the reduced 6MWD improvement induced by the different drugs were three problems that created a lack of power in the conclusions. Savarese et al., [3] showed that in patients with $\mathrm{PAH}$, an improvement in 6MWD did not reflect a benefit in clinical results. The reason for this discrepancy may be the overall shorter clinical follow-up period (a median of 14.5 weeks) and the 34 $\mathrm{m}$ of $\triangle 6 \mathrm{MWD}$, compared to the 6-month follow-up and $65 \mathrm{~m}$ reduction in 6MWD by our study. Of the three studies (using tadanafil with different dosages, imatinib and aspirin) with 24-26 weeks of follow-up [18-20], there was only an average 6MWD improvement of $8 \mathrm{~m}$ in the medication group, which was similar to the $4 \mathrm{~m}$ improvement in the control group. These results are all significantly lower than our findings, which reflect the significant improvement achieved by the PADN procedure.

\section{Clinical Relevance}

The PADN procedure was adapted from previous animal studies $[6,7]$ and was first used in humans in our study [8]. PADN-1 reported a mean 6MWD improvement of $100 \mathrm{~m}$ at 3-months follow-up after the PADN procedure [8], and the current study showed 6-month changes in the 6MWD of $65 \mathrm{~m}$ and a change of $92 \mathrm{~m}$ at one-year after the denervation. Because the PADN procedure was associated with a more significant increase in 6MWD paralleled with the reduction of sPAP and mPAP, and a lower rate of PAH-related events and lower cost compared to previous clinical studies using different drugs [13], demonstrated the clinical importance of PADN for PAH patients.

\section{Limitations}

One major limitation of the current study is the study design. To compensate for this limitation, the control implemented before and after the design support the relevance of the significant improvement shown in 6MWD after PADN compared to using medications. The results confirmed our findings in the PADN1 study [8]. A second limitation is the small sample size in this study, which may not yield results that is generalizable to the general population. Interestingly, we found that when followup was extended to one-year, not only was 6MWD continuously negatively correlated with exercise capacity but also predicted the occurrence of PAH-related events. Furthermore, our results showed that a 6MWD of a minimum of $400 \mathrm{~m}$ might be a good parameter to predict patients' prognosis. Alternatively, this study was conducted at two centers; therefore, a learning curve for PADN should be completed when this procedure is used in other centers. Due to small patient size, we did not perform further comparison between PAH with PH induced by LHD. Lastly, the present study included PAH patients with different etiologies. As a result, our preliminary results should be translated into clinical practice with caution.

\section{Conclusion}

This controlled before and after study showed the improvement of 6MWD and its correlation with exercise capacity and clinical outcomes after the PADN procedure. Further randomized studies with larger patient samples are required to show the benefits of PADN for PAH patients.

\section{Acknowledgments}

We thank Dr. Ling Lin and Ms. Hai-Mei Xu for data collection through the whole study, and all staffs from Central Laboratory of Nanjing First Hospital for the measurement of blood samples. The tough work by Dr. Ling-Ling Zhu and Ms. Jing Kan provided the measurements of 6-minute walk distance at different timing point. We appreciated Dr. Bao-Xiang Duan who served as the Director of Independent Event Committee. We sincerely appreciated Mr.Zhi-Guo Zheng from Pulmo Co.Ltd (Wuxi, China) who provided all PADN devices for the completion of this study. Funding by Jiangsu Provincial Special Program of Medical Science (BL2013001), Nanjing, Jiangsu, China. This study was funded by a grant from the National Natural Science Foundation of China (NSF 81270191 and NSF 91439118).

\section{Supplemantal Data}

\section{Supplemental 1}

1. Supplemental 1.1. Anterior-posterior and cranial (200) view of pulmonary arterial angiograph.

2. Supplemental 1.2. LAO (200) and cranial (200) view of pulmonary arterial angiograph.

3. Supplemental 1.3. PADN catheter in distal main PA.

4. Supplemental 1.4. PADN procedures with ablation by electrode $2-4$.

https://www.youtube.com/playlist?list=PLK59BG1OpHw7zM8P_ qW9Z9VuaxNji_LEn

Supplemental 2. Sympathetic nerves endings of a dog's pulmonary artery mostly localized in the anterior-lateral wall between MPA and LPA.

http://scidoc.org/articlepdfs/IJCRR/IJCRR-2470-4563-03-201_Supplementary. pdf

Supplemental 3. Dynamic change of pulmonary arterial pressure (PAP) in a 42-year patient with IPAH. Systolic PAP and mean PAP decreased from $91 \mathrm{mmHg}$ and $52 \mathrm{mmHg}$ to $79 \mathrm{mmHg}$ and $41 \mathrm{mmHg}$ at 10 -month after PADN. Cardiac echo showed the mean PAP decreased from $42 \mathrm{mmHg}$ at baseline to $30.48 \mathrm{mmHg}$ 48-hour after PADN and 31.3mmHg1-month after PADN. http://scidoc.org/articlepdfs/IJCRR/IJCRR-2470-4563-03-201_Supplementary. pdf 


\section{References}

[1]. Galie N, Hoeper MM, Humbert M, et al., (2009) ESC Committee for Practice Guidelines (CPG). Guidelines for the diagnosis and treatment of pulmonary hypertension: The Task Force for the Diagnosis and Treatment of Pulmonary Hypertension of the European Society of Cardiology (ESC) and the European Respiratory Society (ERS), endorsed by the International Society of Heart and Lung Transplantation (ISHLT). Eur Heart J. 30: 24932537.

[2]. Humbert M, Sitbon O, Chaouat A, et al., (2010) Survival in patients with idiopathic, familial, and anorexigen-associated pulmonary arterial hypertension in the modern management era. Circulation. 122(2): 156-63.

[3]. Galie N, Manes A, Negro L, Palazzini M, Bacchi-Reggiani ML, Branzi A (2009) A meta-analysis of randomized controlled trials in pulmonary arterial hypertension. Eur Heart J. 30(4): 394-403.

[4]. Velez-Roa S, Ciarka A, Najem B, Vachiery JL, Naeije R, van de Borne P (2004) Increased sympathetic nerve activity in pulmonary artery hypertension. Circulation. 110(10): 1308-12.

[5]. Hernandez AF, Hammill BG, O'Connor CM, Schulman KA, Curtis LH, Fonarow GC (2009) Clinical effectiveness of beta-blockers in heart failure findings from the OPTIMIZE-HF Registry. J Am Coll Cardiol. 53(2): 18492.

[6]. Juratsch CE, Jengo JA, Castagna J, Laks MM (1980) Experimental pulmonary hypertension produced by surgical and chemical denervation of the pulmonary vasculature. Chest. 77(4): 525-30.

[7]. Chen SL, Zhang YJ, Zhou L, et al., (2013) Percutaneous pulmonary artery denervation completely abolishes experimental pulmonary arterial hypertension in vivo. EuroIntervention. 9(2): 269-76.

[8]. Chen SL, Zhang FF, Xu J, et al., (2013) Pulmonary artery denervation to treat pulmonary arterial hypertension: the single-center, prospective, firstin-man PADN-1 study (first-in-man pulmonary artery denervation for treatment of pulmonary artery hypertension). J Am Coll Cardiol. 62(12): 1092-100.

[9]. Galiè N, Manes A (2013) New treatment strategies for pulmonary arterial hypertension: hopes or hypes? J Am Coll Cardiol. 62(12): 1101-1102.

[10]. Roberto M. Lang, MD (FASE), Michelle Bierig, MPH, RDCS (FASE), Richard B. Devereux, MD, Frank A. Flachskampf, MD et al., (2005) Recommendations for chamber quantification: a report from the American Society of Echocardiography's Guidelines and Standards Committee and the Chamber Quantification Writting Group. J Am Soc Echocardiogr. 18(12) : 1440-1463.

[11]. Ommen SR, Nishimura RA, Hurrell DG, Klarich KW (2000) Assessment of right atrial pressure with 2-dimensional and Doppler echocradiography: a simultaneous catheterization and cardiographic study. Mayo Clin Proc. 75(1): 24-29.
[12]. Tei C, Nishmura RA, Seward JB, Tajik AJ (1997) Noninvasive Dopplerderived myocardial performance index: correlation with simultaneous measurement of cardiac catheterization measurements. J Am Soc Echocardiogr. 10(2): 169-178

[13]. Savarese GS, Paolillo S, Costanzo P, et al., (2012) Do changes of 6-minute walk distance predict clinical events in patients with pulmonary arterial hypertension? A meta-analysis of 22 randomized trials. J Am Coll Cardiol. 60(13): 1192-1201.

[14]. Benza RL, Miller DP, Gomberg-Maitland M, et al., (2010) Predicting survival in pulmonary arterial hypertension: insights from the Registry to Evaluate Early and Long-Term Pulmonary Arterial Hypertension Disease Management (REVEAL). Circulation. 122(2): 164-72.

[15]. McLauglin VV, Badesch DB, Delcroix M, et al., (2009) End points and clinical trial design in pulmonary arterial hypertension. J Am Coll Cardiol. 54 (1): S97-S107.

[16]. Frost AE, Badesch DB, Miller DP, Benza RL, Melizer LA, McGoon MD (2013) Evaluation of the predictive value of a clinical worsening definition using 2-year outcomes in patients with pulmonary arterial hypertension: a RAVEAL registry analysis. Chest. 144(5): 1521-9.

[17]. McLaughlin VV, Presberg KW, Doyle RL, et al., (2004) American College of Chest Physicians. Prognosis of pulmonary arterial hypertension: ACCP evidence-based clinical practice guidelines. Chest. 126(1): 78S-92S.

[18]. Galie N, Brundage BH, Ghofrani HA, et al., (2009) Pulmonary Arterial Hypertension and Response to Tadalafil (PHIRST) Study Group. Tadalafil therapy for pulmonary arterial hypertension. Circulation. 119(2): 28942903.

[19]. Hiremath J, Thanikachalam S, Parikh K, et al., (2010) TRUST Study Group. Exercise improvement and plasma biomarker changes with intravenous treprostinil therapy for pulmonary arterial hypertension: a placebocontrolled trial. J Heart Lung Transplant. 29(2): 137-49.

[20]. McLaughlin VV, Oudiz RJ, Frost A, et al., (2006) Randomized study of adding inhaled iloprost to existing bosentan in pulmonary arterial hypertension. Am J Respir Crit Care Med. 174(11): 1257-63.

[21]. Mereles D, Ehlken N, Kreuscher S, et al., (2006) Exercise and respiratory training improve exercise capacity and quality of life in patients with severe chronic pulmonary hypertension. Circulation. 114(14): 1482-9.

[22]. Fritz JS, Blair C, Oudiz RJ, et al., (2013) Baseline and follow-up 6-minute walk distance and brain natriuretic peptide predict 2-year mortality in pulmonary arterial hypertension. Chest. 143(2): 315-23.

[23]. Farber HW, Miller DP, McGoon MD, et al., (2014) Predicting outcomes in pulmonary arterial hypertension based on the 6-minute walk distance. J Heart Lung Transplant. 34(3): 362-8.

[24]. Gabler NB, French B, Strom BL, et al., (2012) Validation of 6-minute walk distance as a surrogate end point in pulmonary arterial hypertension trials. Circulation. 126(3): 349-56. 